\title{
ANÁLISIS DE LA SITUACIÓN DE LA SALUD OCUPACIONAL EN CHILE
}

\author{
Olivia Inés Sanhueza Alvarado* \\ Sandra Verónica Valenzuela Suazo** \\ Roxana Verónica Enríquez Quinteros***
}

ALVARADO, O.I.S.; SUAZO, S.V.V.; QUINTEROS, R.V.E. Análisis de la situación de la salud ocupacional en Chile. Rev.latino-am.enfermagem, Ribeirão Preto, v. 7, n. 1, p. 49-54, janeiro 1999.

Las autoras realizan un análisis crítico de la Salud Ocupacional en Chile con base en las conclusiones de un taller multidisciplinario donde participaron profesionales dedicados a esta área y en el cual se utilizó la metodología de planificación estratégica, exponiendo debilidades, fortalezas y sus proyecciones en la salud del ambiente regional. También se discutió un perfil del profesional que debe participar en los programas, se delimitó un plan de trabajo que reflejaría el desarrollo futuro de esta área y resumió el papel de la Universidad en este plan de trabajo, se analizó el futuro de la Salud Ocupacional.

TÉRMINOS CLAVES: salud ocupacional, enfermería ocupacional, equipo de salud interdisciplinario, trabajador, seguro nacional de salud

\section{INTRODUCCIÓN}

En América Latina el desarrollo de la Salud Ocupacional (S.O.) se remonta a la década del veinte con los primeros intentos de protección a los trabajadores. En Chile, desde 1924, a través de la ley 4054, la legislación estableció la responsabilidad del empleador en la génesis de los accidentes y enfermedades profesionales de sus empleados, pero no obligaba al pago de una cotización que cubriese esa responsabilidad generando la posibilidad de su ejercicio efectivo por parte de los trabajadores.

Como informa SANDOVAL et al. (1994), esto llevó a que sólo las grandes empresas, o aquellas con sindicatos fuertes, que hacían valer sus derechos contrataban seguros contra accidentes del trabajo y enfermedades profesionales. La gran mayoría de los trabajadores tenía que plantear un litigio ante los tribunales del trabajo con el fin de obtener el reconocimiento de sus derechos, lo que en la práctica imposibilitaba ejercerlos.

El sistema de seguros con compañías privadas al no ser obligatorio se mostró ineficiente tanto, por los costos que suponía para los empleadores como por la mala atención que otorgaba a los trabajadores accidentados y enfermos. Esto condujo a que los empleadores se organizaran para crear sus propios seguros en forma de mutuales sin fines de lucro, que nacieron antes de la vigencia de la ley actual, (SANDOVAL et al., 1994).

A partir del año 1947 un grupo de expertos organiza servicios de S.O. en varios países sudamericanos. En junio de 1963, en convenio con la OMS, se crea el Instituto de Higiene del Trabajo y Contaminación Atmosférica de Santiago. La legislación chilena contempló la teoría del riesgo profesional en materia de accidentes del trabajo y enfermedades profesionales hasta el 30 de abril de 1968, ya que a partir del $1^{\circ}$ de mayo de ese año entró en vigencia la Ley $\mathrm{N}^{\circ} 16.744$ (CHILE, Ley 16.744), que adoptó la teoría de riesgo social, creando un seguro social contra riesgos de accidentes del trabajo y enfermedades profesionales (GIACONI, 1995).

Esta ley establece un Seguro Social obligatorio, de carácter integral, que tiene por objeto prevenir la ocurrencia de accidentes del trabajo y enfermedades profesionales, otorgar prestaciones médicas y pecuniarias en caso de suspensión, disminución o terminación de la capacidad de ganancia o muerte del trabajador y rehabilitarlo tanto en su capacidad física y síquica como profesional, de modo de posibilitar su reinserción al trabajo.

Según MOLINA (1977), durante el siglo XX fueron las consideraciones económicas más bien que las humanitarias las que mejoraron las condiciones de trabajo.

\footnotetext{
* Profesora Asociada, Doctoranda en Enfermería Programa Interunidades, Escuela de Enfermría de Ribeirão Preto, Docente del Dpto. de Enfermería, Facultad de Medicina, Universidad de Concepción, Chile

** Profesora Asistente, Doctoranda en Enfermería Programa Interunidades, Escuela de Enfermería de Ribeirão Preto, Docente Dpto. de Enfermería, Facultad de Medicina, Universidad de Concepción, Chile

*** Profesora Asociada, Docente Dpto. de Enfermería, Facultad de Medicina, Universidad de Concepción, Chile
} 
Las nuevas y cada vez más complejas industrias estimularon el surgimiento de la especialización de los obreros y una fuerza de trabajo más difícil de reemplazar. La organización de los trabajadores apoyó los movimientos sociales de los siglos XIX y XX, iniciando sus justas conquistas laborales por un medio ambiente laboral más adecuado.

La Ley vigente, que regula la materia sobre accidentes del trabajo y enfermedades profesionales tiene casi 30 años. En Enfermería reconocemos que existe un importante desconocimiento con respecto a esta materia, aún cuando formemos parte del equipo que participa en la implementación de la ley, en organismos administradores como son las Mutuales, las empresas con Administración Delegada y en el Servicio de Salud.

Se estima que en la preparación académica de los estudiantes de Enfermería en Chile existe un vacío con respecto a estas materias o si se entregan, son escasas. Cursos especializados con respecto a la Salud del Trabajador no son frecuentes ni expeditos y en su organización es además, poco frecuente encontrar enfermeras participando, ya que este profesional no tiene una especialización formal y reconocida en la materia, sólo aquella que otorga el ejercicio diario de su quehacer en la industria, mutual u otra entidad.

El presente trabajo nació por las motivaciones anteriores y con la convicción de que estamos iniciándonos en una temática que relaciona al hombre y a su medio ambiente, como otras en Enfermería, en este caso el trabajador y su ambiente de trabajo, área que consideramos no es exclusivo del quehacer de este profesional, sino que es compartido multidisciplinariamente por otras disciplinas, como son la ergonomía, psicología, ingeniería, medicina, derecho, sociología y otras.

Para apoyar un análisis de la situación actual de la S.O. en Chile, organizamos en el mes de marzo del presente año en el Departamento de Enfermería de la Universidad de Concepción, en la ciudad de Concepción, un Curso Seminario en Salud Ocupacional, donde participaron profesionales ligados a empresas, hospitales y universidad. Estos, en su mayoría dedicados a la Salud Ocupacional en sus respectivas instituciones, correspondiendo su actividad profesional a ingenieros, enfermeras, médicos y expertos en prevención de riesgos. Se realizó un taller de trabajo grupal, los participantes se dividieron en tres grupos. La metodología utilizada fue la "planificación estratégica" y el propósito fue:

- "Analizar multidisciplinariamente la situación de programas de salud ocupacional existentes actualmente en Chile".

\section{PROPÓSITO}

"Analizar la situación de la salud ocupacional en Chile, desde una perspectiva de los resultados de Taller en Salud Ocupacional".

\section{OBJETIVOS}

Los objetivos de este trabajo fueron los siguientes: 1. Analizar las fortalezas, debilidades y proyecciones de los Programas de Salud Ocupacional actuales.

2. Determinar el perfil del profesional que debe participar en estos programas.

3. Analizar el futuro de la Salud Ocupacional y sus proyecciones en la Salud Ambiental de la región.

4. Delimitar un plan de trabajo que refleje el desarrollo futuro de la Salud Ocupacional.

5. Resumir como se visualizaría el papel de la Universidad en este plan de trabajo.

A continuación se presentan los resultados a los cuales llegaron los grupos en el trabajo de taller, finalizando cada uno de ellos con un análisis y consideraciones realizadas por las autoras del presente trabajo.

\section{RESULTADOS Y ANÁLISIS DEL TRABAJO EN EL TALLER GRUPAL}

\section{A. 1. Fortalezas de los programas de S.O. actuales}

Los grupos estimaron lo siguiente:

- Existe una Ley de Accidentes del Trabajo y Enfermedades Profesionales, $N^{\circ} 16.744$, la que protege al trabajador.

- Existe un cambio cultural en los problemas del medio ambiente.

- Existe calidad de atención (de excelencia), entregada por las Mutuales u otras entidades administradoras de la ley.

- Existencia de programas establecidos.

- Existen recursos humanos, técnicos y económicos.

- Existe un equipo multidisciplinario.

- Existencia de trabajo en equipo y motivación del grupo.

- Existencia de apoyo gubernamental y empresarial, aunque no existe un conocimiento acabado.

- Existen organismos asesores que prestan apoyo directo a las empresas, sin ánimo sancionador.

Consideramos que si bien existe una "ley que protege al trabajador" esta es muy ambigua frente a algunos problemas de salud actuales como por ejemplo los problemas relacionados con el stress laboral, ya que se le puede dar diversas interpretaciones. Los recursos 
mencionados son variados y se disponen principalmente de ellos en aquellas administradoras como son las Mutuales y en empresas grandes con Administración Delegada, situación que no es igual a nivel del Instituto de Normalización Previsional/Sistema Nacional de Servicios de Salud (S.N.S.S.) del Estado. Estos, además de ser organismos administradores de esta ley otorgan las prestaciones médicas en esta área a la población desprotegida calculada en 1.641 .400 personas, esto implica que estos servicios tienen la responsabilidad de realizar acciones de Salud Ocupacional sobre el 49,8\% de la fuerza de trabajo ocupada en el país, sin mencionar que el sector estatal presentaría además graves problemas de calidad y oportunidad, (SANDOVAL et al., 1994). En el año 1993 la población protegida por la ley $\mathrm{N}^{\circ} 16.744$, alcanzó a un $65,8 \%$ de la fuerza de trabajo ocupada; por lo que los trabajadores desprotegidos serían un $34,2 \%$.

Según DÍAZ (1994), esta población desprotegida estaría constituida por un importante porcentaje de mujeres que trabajan por periodos, como son las trabajadoras que laboran durante la temporada de recolección de frutas, las "temporeras" en la agricultura, las asesoras del hogar, las que se dedican a la manufactura y recolección de materiales para su reciclado. Esto trae consigo problemas para los trabajadores que no pueden acogerse a los beneficios de la ley y generan un subregistro ya que los accidentes y enfermedades profesionales de estas personas no son notificados como corresponde en las diversas entidades administradoras.

Según SANDOVAL et al. (1994), la experiencia del Seguro Social de Accidentes del Trabajo y Enfermedades Profesionales ha sido hasta hoy día exitosa en cuanto a su capacidad para crear servicios médicos especiales para casi el $50 \%$ de la fuerza de trabajo ocupada que está afiliada al sistema de mutualidades que ha permitido concretar recursos para beneficio directo de los trabajadores, lo que aparece claro para la atención médica. También se ha podido generar estructuras y actividades para la prevención de riesgos cuya eficacia aparece mucho menos clara que la alcanzada en relación a la atención médica.

Esta situación es contrastada con la fracción de la población ocupada que teniendo cobertura a través del Instituto de Normalización Previsional/Sistema Nacional de Servicios de Salud (S.N.S.S.) no tienen los servicios diferenciados del resto de la población beneficiaria del S.N.S.S., con todos los elementos negativos que esto significa.

Aunque en las conclusiones se menciona la existencia de un equipo multidisciplinario pensamos que este equipo esta ausente en la mayoría de las entidades de salud ocupacional, sólo se observaría nominalmente, funcionando en las Mutuales y en algunos servicios de Administración Delegada.
A. 2. Debilidades de los programas de S.O. actuales

Los grupos llegaron a las siguientes conclusiones: - Falta de claridad legislativa de la ley existente.

- No existen asesores legislativos que permitan mejorar la ley.

- Ausencia de postgrados en S.O.

- Déficit de conocimientos preventivos desde niveles básicos de la población.

- A nivel universitario existe escaso aporte en preparación formal de profesionales en S.O.

- Desconocimiento de la ley 16.744 por parte de los usuarios y pequeños empresarios.

- Falta de un equipo multidisciplinario.

- Existen problemas de comunicación y coordinación entre organismos administradores de la ley, empresa y trabajador.

- Falta de actualización de la ley en cuanto a identificación de riesgos.

- Fallas o vacíos legales en la legislación actual.

- Centralización de los niveles de decisión y de fiscalización, lo que retrasa las resoluciones de los casos presentados en la Comisión de Pensiones e Invalidez (COMPIN).

- Falta de recursos humanos, técnicos y profesionales para la labor de S. O.

Observamos respuestas contradictorias en estas conclusiones a las cuales llegaron los grupos, ya que en el anterior, se reconoce la existencia de un equipo multidisciplinario, de programas en S.O. y la disponibilidad de recursos tanto humanos como los de infraestructura, todos los cuales son negados en este punto.

Creemos que esto traduce, tal como se ha mencionado, un conocimiento incompleto de la situación actual. También la crítica que se le realiza a la ley es parcial ya que se aprecia el desconocimiento de ella, reconociéndose si su ambigüedad y su escaso análisis, que consideramos debiera ser permanente para el perfeccionamiento de la ley por las personas que trabajan en S. O. y que participaron en este taller.

Consideramos que en relación al problema de las asesorías que realizan algunas instituciones de salud ocupacional a las mismas empresas que tienen afiliadas, se advierte, tal como lo señalara un estudio sectorial realizado por GRASSET (1995), la necesidad de que exista un Consejo Nacional de Salud Ocupacional, a fin de asesorar en estas materias a las autoridades de los ministerios del Trabajo y Previsión Social y de Salud; recomendar políticas en salud ocupacional; estudiar y proponer las modificaciones legales y reglamentarias que se estimen pertinentes introducir; analizar las situaciones que se presenten a su conocimiento y desempeñar todas las demás funciones que le indiquen las leyes. Este Consejo no tendría facultades decisorias, según señala en su análisis el autor mencionado, sino que simplemente 
asesora, con lo cual facilitaría la adopción de políticas nacionales en salud ocupacional, lo que actualmente se echa de menos.

Para lograr sus objetivos, el Consejo debería estar integrado por representantes de las más diversas áreas y actividades relacionadas con el régimen de riesgos laborales, tales como de los ministerios de Salud y del Trabajo y Previsión Social; de las universidades, de los trabajadores, de los empleadores, de las Mutualidades de Empleadores y de los expertos en prevención de riesgos.

\section{A. 3. Proyecciones de la salud ocupacional}

Los grupos consideraron las siguientes:

- Son amplias y diversas para proteger al trabajador, lo que redundará en el mejoramiento de las condiciones laborales.

- La toma de conciencia que se vislumbra respecto al cuidado del medio ambiente debe dar frutos par una mejor calidad de vida junto a proyecciones económicas.

- Se implementarán los programas necesarios, se tratará de mejorar lo que existe ampliando la cobertura para cubrir las necesidades reales.

- Hay un convencimiento de que si no hay cambios se producirá un estancamiento.

En este punto tenemos la certeza de que en Chile la S.O. aún es un campo escasamente explorado del cual recién se están descubriendo sus trascendentales implicanciones, ya que se ha dado por hecho durante mucho tiempo el siguiente párrafo:

"Nunca he visto a un herrero actuando como embajador o a un fundidor enviado a alguna misión; pero he visto al obrero metalúrgico en el trabajo, encadenado a la boca del horno, o al albañil, expuesto a todos los riesgos e inclemencias del tiempo", tomado de los Papiros Egipcios de Sellier apud MOLINA (1977).

La historia nos muestra que la vida del trabajador no ha sido fácil, tal como se reseña en los primeros registros de Salud Ocupacional en la obra de Bauer, G. "De Re Metallica", publicada en 1556, donde se señala la escasa expectativa de vida de los mineros pues se había encontrado en los Cárpatos a mujeres viudas de siete maridos; pasando por la revolución industrial en el siglo XVIII y la posterior lucha de los trabajadores durante los siglos XIX y XX, donde se lograron justas conquistas laborales por un medio ambiente más adecuado, (MOLINA, 1977).

Creemos que esto ya está en un proceso de cambio positivo, desde el "darse cuenta" del tiempo perdido hasta que el Estado, la Empresa Privada y la Universidad e Institutos Técnicos por su rol formador de futuros profesionales comiencen a participar en el cambio, reforzando la creación de un profesional con mentalidad futurista, flexible, respetuosos de las personas y por lo tanto de los trabajadores y del ambiente.

\section{B. El perfil del profesional de S.O.}

Los grupos destacaron que debería poseer las siguientes características:

- Sea especialista y profesional

- Líder, para que motive

- Comprometido con su labor, que esté convencido

- Que tenga una preparación formal

- Que pertenezcan a diferentes áreas

- Su postura profesional deberá permitir credibilidad en él

- Ser modelo para la gente con la que trabaja.

- En capacitación continua

- Ser educador innato

- Tenga vocación

- Sea comunicativo

- Amplitud de criterio

- Creativo

- Flexible

- Buena llegada a diferentes niveles

- Empático

- Con capacidad de adaptación frente a diferentes situaciones

Con respecto a estas características concordamos en todos sus aspectos con las cualidades mencionadas, en especial, consideramos que es de extraordinaria importancia que sea especialista en el área, trabaje en equipo y tenga buena llegada a diferentes niveles, aún cuando, las otras características no se manifiesten con la misma intensidad en un mismo profesional, no olvidando que durante el desempeño de su trabajo no lo hace individualmente sino integrado en un equipo de personas con diferentes cualidades y características que se complementan entre sí.

\section{Futuro de la S.O. y sus proyecciones en la salud ambiental}

Los grupos llegaron a las siguientes conclusiones: - "Llegar a ocupar el lugar que le corresponde a partir de la toma de conciencia de derechos y obligaciones de los diferentes niveles: trabajador-empresa-organismos administradores".

- "Énfasis en la Prevención de Riesgos a todo nivel, para llegar a un ambiente laboral libre de ellos, (sociedad con riesgos mínimos)".

- "Al preocuparnos de la S. O., de la Salud Ambiental y de la calidad de vida mejorarán notablemente, lo que permitirá que como unidades biopsicosociales mantendremos un equilibrio más adecuado".

También compartimos estas conclusiones, ya que vemos al trabajador en un futuro cercano participando activamente, organizado, conocedor y defensor de sus 
derechos. Vemos a empresarios con una mentalidad más humanista y visionarios. Consideramos que estos cambios no serán fáciles ni rápidos, requerirán de personas no sólo con respeto por ellas mismas, sino también respeto por el medio ambiente.

\section{Plan de trabajo en salud ocupacional}

Los grupos consideraron lo siguiente:

- "Se deben establecer programas, difundirlos y ejecutarlos, considerando los siguientes factores: salud; integridad física de los trabajadores; conservación del medio ambiente y patrimonio de la empresa".

- "Conseguir apoyo gubernamental que permita legislar sobre programas educativos a diferentes niveles, que permitan la especialización profesional en S.O.”.

- "Constituir una red de apoyo multidisciplinaria que permita una retroalimentación constante en las materias de S.O."

- "Trabajo mancomunado e interdisciplinario, donde están reflejados todos los estamentos de la sociedad, con profesionales de todas las áreas, de todos los niveles, sexo y edad".

Estamos ciertos que por muchos años la tendencia de los Programas de Salud en Chile, y no sólo en S.O., sino que en general, han sido con una perspectiva curativa dejando de lado las acciones preventivas. Actualmente se aprecia en los programas generales un cambio hacia la prevención, lo cual también es lógico que debiera ocurrir con S.O.

La necesidad de promover y proteger la salud de los trabajadores será cada vez más imperiosa a medida que el desarrollo económico avanza y requiere de una fuerza de trabajo más especializada. Este hecho obliga, como lo señala MOLINA (1977), a la formulación de programas de higiene y seguridad y de vigilancia epidemiológica tanto de la exposición a agentes presentes en el lugar de trabajo, como del daño a la salud. Además se deberá incorporar el concepto de salud integral del trabajador para considerar las interacciones entre los riesgos y las patologías consideradas de enfermedad común y los riesgos y patologías considerados laborales.

\section{E. PAPEL DE LA UNIVERSIDAD}

Los grupos destacaron que la Universidad debe ser:

- "Ente formador e integrador, con extensión hacia la sociedad".

- "Que forme al especialista que lidera al equipo".

- "Incluir el tema de S.O. en los curriculum de las carreras".

- "Vemos una ausencia de estudios de S.O. liderados por la Universidad.
- "Ausencia de entrega de asignaturas en los niveles de pre y postgrado.

- "En el futuro, deberá cumplir un rol importante que permita realizar especializaciones o postgrado en S.O., creando previamente la inquietud en el pregrado sobre el tema.

- "Los profesionales que trabajan en el área cuenten con la facilidad de ser nivelados para la obtención del grado." - "Cursos de Perfeccionamiento en el área de tipo multidisciplinarios".

Tal como lo señaláramos en un comienzo, reconocemos que el tema de S.O. ha sido dejado de lado por muchos años en Chile. Los cambios estructurales implementados a comienzos de los años 80 , tanto en las áreas de salud como trabajo y previsión social, si bien en lo formal mantuvieron casi inalteradas las estructuras y normas del sistema de salud ocupacional, en la práctica ellas contribuyeron a que se desdibujaran las diversas funciones que la Ley 16.744 asignara al estado en este sector, (SANDOVAL et al., 1994).

En la década del 70 también como producto de drástica disminución del gasto público, producto de la recuperación económica, las instituciones del área ya habían experimentado una muy fuerte restricción en sus recursos humanos, materiales e institucionales.

Visualizamos actualmente un renovado interés por todos los temas relacionados al área de la salud del trabajador. Creemos que se debe principalmente a la relación que existe entre ambiente de trabajo y medio ambiente, concepto este último que en todo el mundo se ha visto cuestionado, dada la problemática de contaminación actual existente. Un ejemplo a nivel nacional de esta preocupación es el Instituto de Salud Pública de Chile quien, según informa SANDOVAL et al. (1994), ha cumplido una labor fundamental en los últimos 10 años con los estudios clínicos epidemiológicos de "arsenicismo" en trabajadores de las fundiciones de Cobre; la exposición profesional a radiaciones ionizantes y el trauma acústico como factor de incapacidad laboral.

En los últimos años y posterior a la creación de la Comisión de Descontaminación de la Región Metropolitana, se ha dado un mayor énfasis a los temas de contaminación ambiental urbana por las serias implicancias a nivel de la salud humana.

Asimismo, observamos que la Universidad tampoco se ha quedado atrás en los temas relacionados con el medio ambiente, ya que existen Programas y Proyectos muy importantes que se han ido desarrollando. Pero, aún es insuficiente su aporte en los curricula de las diferentes carreras que imparte. Recientemente en la Universidad de Concepción se han comenzado a implementar asignaturas electivas, multidisciplinarias, que están enfocando la salud del trabajador desde un punto de vista multisectorial y factorial. 
CONSIDERACIONES FINALES

El conocimiento y la reflexión de estos hechos durante estos últimos años han permitido a las autoras darse cuenta de la enorme trascendencia que tiene para la vida de las personas la preocupación de las condiciones laborales y ambientales de los trabajadores.

Ha cambiado de una concepción de "Medicina Ocupacional" hacia "Salud Ocupacional", lo cual es un enorme cambio por todo lo que esto trae consigo. Enfermería entonces tiene una responsabilidad activa y no sólo como participante de las acciones médicas que se realicen por la salud del trabajador.
Pensamos que formamos parte de un equipo multidisciplinario que actúa para favorecer la salud de los trabajadores, y creemos que Enfermería, dada su preparación, conocimiento y cualidades organizativas, tiene la posibilidad de liderar el trabajo en el "Equipo de Salud Ocupacional”.

Nos queda mucho por delante, tenemos enormes desafíos, como es obtener un reconocimiento en la Especialización en Salud Ocupacional, debemos estar en los espacios de toma de decisiones, hacer oír nuestras opiniones, y todo ello se podrá lograr con mayor estudio, análisis y preparación de todos los profesionales de Enfermería cuyo propósito es mejorar la calidad de vida de las personas y a la vez mejorar las del medio ambiente.

\section{ANALYSIS OF THE SITUATION OF OCCUPATIONAL HEALTH IN CHILE}

The authors make a critical analysis of the Occupational Health in Chile based on the conclusions obtained from a multidisciplinary workshop, attended by professionals of this area. The method used was strategic planning, exposing the weaknesses, strengths and expectations regarding the health reality of the region. A profile of the type of professionals that should participate in the programs was also discussed, elaborating a work plan that should reflect the future development in this area, as well as pointing out the role of the University in the work plan and analysing the future of Occupational Health.

KEY WORDS: occupational health, occupational nursing, worker, interdisciplinary health team, national health insurance

\section{REFERENCIAS BIBLIOGRÁFICAS}

01. CHILE. Ley 16.744. Seguro Social contra riesgos de Accidentes y Enfermedades Profesionales. MUSEG C.CH.C. 1968. 159 p.

02. DÍAZ, X. Propuestas en el campo de la salud ocupacional de las trabajadoras en Chile. Santiago, 1994. 81p.

03. GIACONI, J. La salud en el siglo XXI: cambios necesarios. Santiago: Centro de Estudios Públicos (CEP), 1995.
04. GRASSET, A. Salud ocupacional en Chile. In: GIACONI, J. La salud en el siglo XXI: cambios necesarios. Santiago: Centro de Estudios Públicos (CEP), 1995. p. 129-148.

05. MOLINA, G. Introducción a la salud pública: fomento y promoción de la salud ocupacional. Medellín: Escuela Nacional de Salud Pública, 1977.p.107-116.

06. SANDOVAL, $H$. et al. Tercer informe de avance proyecto fortalecimiento del sistema de salud ocupacional en Chile. Santiago: Banco MundialMinisterio de Salud, 1994. 162 p. 